\title{
Urinary prostasin in normotensive individuals: correlation with the aldosterone to renin ratio and urinary sodium
}

\author{
Oliviero Olivieri ${ }^{1}$, Laura Chiecchi ${ }^{1}$, Francesca Pizzolo ${ }^{1}$, Annalisa Castagna ${ }^{1}$, Ricciarda Raffaelli ${ }^{2}$, \\ Muthukumar Gunasekaran ${ }^{1}$, Patrizia Guarini ${ }^{1}$, Letizia Consoli ${ }^{1}$, Gianluca Salvagno ${ }^{3}$ and Kenichiro Kitamura ${ }^{4}$
}

Prostasin, a glycosylphosphatidylinositol (GPI)-anchored serine protease, activates the epithelial sodium (Na) channel (ENaC), and prostasin is released in extracellular fluids, including urine. Previous data have suggested a direct association between urinary prostasin and the activation of an aldosterone-driven pathway, but a quantitative association has never been demonstrated in normotensive subjects. Similarly, physiological relationships with natriuresis or possible gender- or female hormone-related changes in urinary prostasin concentrations have never been investigated. We measured urinary prostasin by enzyme-linked immunosorbent assay in $\mathbf{4 3}$ healthy normotensive subjects of similar age presenting different urinary $\mathrm{Na}$ levels and in 15 women during the menstrual cycle and after oral estro-progestinic contraceptive (OC) therapy. Exosomal urinary prostasin was also estimated by western blotting of samples from six healthy subjects twice during the morning. Urinary prostasin presented a wide range of values (from 0.5 to $18.9 \mathrm{~nm}$ ) without gender differences. It was positively correlated with the aldosterone to renin ratio (ARR) but not with circulating aldosterone or renin individually. Urinary prostasin was directly correlated with U-Na levels (up to $200 \mathrm{nmol} \mathrm{Na}$ ), whereas it decreased for higher $\mathrm{Na}$ concentrations. In women, no significant changes of prostasin concentration were observed during menstrual phases. After $\mathrm{OC}$ therapy, prostasin increased (from $2.37 \pm 1.27$ to $4.85 \pm 5.28 \mathrm{~nm})$, although the increase was not statistically different $(P=0.07)$. Prostasin was detectable in urinary exosomes and displayed a pattern similar to urinary prostasin in relation to urinary $\mathrm{Na}$. In conclusion, urinary prostasin correlates with the ARR, and it is physiologically modulated by natriuresis in normotensive individuals.

Hypertension Research (2013) 36, 528-533; doi:10.1038/hr.2012.232; published online 24 January 2013

Keywords: ARR; natriuresis; prostasin

\section{INTRODUCTION}

Aldosterone, the major mineralocorticoid hormone in humans, has a pivotal role in maintaining sodium $(\mathrm{Na})$, maintaining water body balance and ultimately in regulating blood pressure (BP) mainly through the modulation of $\mathrm{Na}$ reabsorption at the kidney level. In particular, an aldosterone-driven pathway leads to the activation of the epithelial $\mathrm{Na}$ channel $(\mathrm{ENaC})$ at the distal tubule and collecting duct level, finally promoting cell $\mathrm{Na}$ entry for potassium $(\mathrm{K})$ exit in equimolar proportions. The $\mathrm{ENaC}$ is a membrane complex localized not only in the kidney but also in several other tissues and organs involved in $\mathrm{Na}$ maintenance (that is, airways, skin, colon and salivary glands). The channel is composed of three homologous subunits $(\alpha, \beta$ and $\gamma)^{1}$ that are functionally complementary in activating the ENaC by a sequential mechanism of co-operation. These subunits are only partially recognized and characterized. ${ }^{2}$

In recent years, great attention has been specifically devoted to proteolytic mechanisms able to activate the $\mathrm{ENaC}$ by removing inhibitory peptides on the $\beta$ or $\gamma$ chains. One of the most interesting proteins investigated in this context is prostasin, a $40-\mathrm{kDa}$ serine protease first detected in the prostate gland and seminal fluid. ${ }^{3,4}$

Prostasin activates ENaC when expressed in oocytes, and the addition of aldosterone to cultured mouse collecting ducts increases $\mathrm{ENaC}$ activity by increasing prostasin secretion, mRNA and protein expression. ${ }^{5,6}$ In conclusion, the currently available data consistently suggest that aldosterone induces the activation of prostasin and, in co-operation with other protease(s), such as furin, cleaves (at one or more sites) the $\gamma$-subunit of $\mathrm{ENaC}$, contributing to the removal of an inhibitor peptide and increasing the probability of the channel opening. ${ }^{7,8}$ Prostasin is also of interest because it is a glycosylphosphatidylinositol (GPI)-anchored protein, secreted in extracellular fluids such as urine, ${ }^{9}$ and the amount released in urine seems to be grossly proportional to the extent of ENaC activation. ${ }^{6}$ Narikiyo et al. $^{6}$ were the first to demonstrate an overexpression of prostasin in the urine of three patients affected by Conn's adenoma,

${ }^{1}$ Department of Medicine, Unit of Internal Medicine B, University of Verona, Verona, Italy; ${ }^{2}$ Section of Gynecology and Obstetrics, Department of Life and Reproduction Sciences University of Verona, Verona, Italy; ${ }^{3}$ Section of Clinical Chemistry, Department of Life and Reproduction Sciences, University of Verona, Verona, Italy and ${ }^{4}$ Department of Nephrology, Kumamoto University, Kumamoto, Japan

Correspondence: Professor O Olivieri, Department of Medicine, Unit of Internal Medicine B, University of Verona, Policlinico GB Rossi, Piazzale LA Scuro 10, Verona 37134, Italy. E-mail: oliviero.olivieri@univr.it

Received 24 September 2012; revised 9 November 2012; accepted 15 November 2012; published online 24 January 2013 
which dramatically decreased after adrenal surgery, thus suggesting a link between abnormal aldosterone secretion and the release of prostasin in urine.

In a previous study, ${ }^{10}$ we demonstrated, for the first time, that prostasin is detectable in the urine of healthy subjects of both genders (that is, regardless of the presence of a prostate). Furthermore, we demonstrated that after anti-aldosterone therapy with spironolactone, urinary prostasin decreased in subjects with low $\mathrm{Na}$ intake (in which the renin-aldosterone axis was activated), while it was unchanged in subjects with a high $\mathrm{Na}$ intake. We hypothesized that there is a basal amount of prostasin, constitutively released in urine regardless of $\mathrm{Na}$ intake and hormone activation, and an 'aldosterone-responsive' aliquot, strictly influenced by aldosterone and $\mathrm{Na}$ balance.

However, these preliminary results, which were based on immunoblotting methods, did not provide a precise quantification of the absolute prostasin concentration. This was achieved for the first time in 2009 by two independent groups that described a newly developed radioimmunological method to assay prostasin in human urine; ${ }^{11,12}$ however, limited data are currently available regarding the physiological variability and regulation of prostasin release in urine.

It is well known that in women, sex hormone fluctuations during the menstrual cycle are associated with corresponding variations of the renin-aldosterone axis. ${ }^{13}$ In a recent study, we demonstrated that both natural and pharmacological female hormones, such as those contained in oral contraceptive (OC) pills, influence aldosterone and renin concentrations and affect the diagnostic work-up for primary aldosteronism. ${ }^{14}$ It has been recently reported that prostasin is expressed at a relatively high level in human placenta trophoblasts in early pregnancy weeks, ${ }^{15}$ further supporting a possible correlation with sex hormones.

As physiological cyclic variations of female hormones alter $\mathrm{Na}$ balance and affect salt retention, we hypothesize that urinary prostasin might be accordingly modulated during different phases of the menstrual cycle and/or after OC therapy. Exosomes are small $(20-100 \mathrm{~nm})$ membrane vesicles that originate as internal vesicles of multivesicular bodies and are released into the extracellular environment (including urine) by the fusion of multivesicular body outer membranes with the plasma membrane. Therefore, exosomes represent a good source of proteins of tubular cell origin. ${ }^{16}$

Based on all of these considerations, we planned this study with the following aims:

(i) to define possible gender-specific differences in urinary prostasin concentrations between males and females of similar age, in order to definitively rule out the influences of possible spillage from the prostate gland, the major source of prostasin in humans;

(ii) to study the relationships between the concentration of urinary prostasin, natriuresis and the renin-aldosterone axis markers of activation;

(iii) to assess the concentration of urinary prostasin in healthy women, during the different phases of the menstrual cycle, in order to evaluate possible changes in parallel with aldosterone and renin variations; and

(iv) to similarly explore the OC therapy effects on prostasin concentration.

Finally, to confirm the data obtained from the urine, natriuresisassociated changes of 'cell' prostasin were also investigated in urinary exosomes. In agreement with a recent study on animal models reported by van der Lubbe et al., ${ }^{17}$ prostasin was detectable in human urinary exosomes.

\section{METHODS}

\section{Healthy subjects}

We enrolled 43 normotensive individuals ( 15 women and 28 men) among the healthy volunteers the Center of Clinical Research of the 'Azienda OspedalieroUniversitaria Integrata' of Verona, Italy. The volunteers consumed an unrestricted diet, had no personal or familial history of hypertension or other significant diseases, and presented normal BP values; moreover, a criterion for enrollment was that the subjects had preliminary routine blood tests (including serum creatinine) in the normal range. Female subjects had to have a negative pregnancy test and regular, predictable, cyclic menses in order to avoid menstrual bleeding during the study period $(48 \mathrm{~h})$. After informed consent was obtained, each subject was asked to collect his or her first morning urine. Plasma $\mathrm{Na}^{+}, \mathrm{K}^{+}$, creatinine, aldosterone and active renin levels were assayed in venous blood samples drawn after overnight fasting.

\section{Healthy fertile women on free and OC-induced menstrual cycles}

Fifteen young healthy normotensive women, with no history of disease and referred for contraceptive counseling to a single physician at the Obstetric and Gynecology Department of the University of Verona, were enrolled in the study. Urine and blood samples were collected in the follicular phase of their menstrual cycles (between the 14th and 16th day), in the luteal phase (between the 20th and 22nd day) and after 2 months of OC therapy. All patients prescribed the same third-generation pill, which consist of $0.075 \mathrm{mg}$ gestodene and $0.02 \mathrm{mg}$ estradiol. At each of the three time points, BP was measured and the average values were recorded.

The protocol was approved by our institutional review committee, and written informed consent was obtained from each subject before initiation of the study.

Systolic and diastolic BP were carefully measured in all enrolled study subjects. BP was evaluated twice in both arms, with the subject remaining in the sitting position for $5 \mathrm{~min}$ and the subject avoiding coffee intake and smoking during the preceding $30 \mathrm{~min}$. Further measurements (when necessary) were performed if the differences in $\mathrm{BP}$ values were $\geqslant 5 \mathrm{~mm} \mathrm{Hg}$.

\section{Prostasin assay}

A complete protease inhibitor cocktail (Roche Diagnostics, Basel, Switzerland) was added to the freshly collected urine, and the $\mathrm{pH}$ was adjusted to 7.0 by adding $1 \mathrm{~m} \mathrm{NaOH}$ as needed. Urine samples were centrifuged at $4{ }^{\circ} \mathrm{C}$ and then filtered and finally concentrated (see below). The samples were stored at $-30{ }^{\circ} \mathrm{C}$ until use.

A competitive enzyme-linked immunosorbent assay (ELISA) test was set up by exploiting the rabbit response toward a specific, highly immunogenic, prostasin peptide (amino sequence: AHQLDSYSEDAKVSTLKDI). Polyclonal antibodies were obtained from rabbits inoculated according to the method previously described by Koda et al. ${ }^{11}$ In brief, antiserum was generated in a rabbit by means of a lymph node injection technique. After 2 weeks, blood was drawn and the serum was separated and stored. After specific purification, the antibody was neutralized, lyophilized and reconstructed; ${ }^{11}$ no cross-reactivity with kallikrein, renin, angiotensin-converting enzyme, prostaglandins and steroids, was demonstrated by the antibody. ${ }^{11}$ The assay details are also part of a patent (US2009/0170126Al, 2 July 2009). Briefly, microtiter plates were coated with purified specific anti-prostasin antibodies dissolved in coating solution $\left(2 \mathrm{mg} \mathrm{ml}^{-1}\right.$ antibody, $100 \mu \mathrm{l}$ per well $)$ by incubation for $16 \mathrm{~h}$ at $4{ }^{\circ} \mathrm{C}$. Each plate was washed three times with phosphate-buffered saline with Tween20 (PBST; Tween 0.5\%) and was blocked with bovine serum albumin (1\%) for $2 \mathrm{~h}$ at room temperature. Serial dilutions of prostasin peptide and aliquots of the urine samples $(50 \mu \mathrm{l}$ per well) along with biotinylated prostasin peptide $\left(2.5 \mathrm{ng} \mathrm{ml}^{-1}, 50 \mu \mathrm{l}\right.$ per well) were dispensed into the coated plate and incubated for $1 \mathrm{~h}$ on an orbital rotating shaker at room temperature. Subsequently, the plate was washed three times with phosphate-buffered saline with Tween-20, and avidin peroxidase conjugates (1/20000 with diluent, $100 \mu \mathrm{l}$ per well) were added. This step was followed by incubation for $1 \mathrm{~h}$ on an orbital rotating shaker at room temperature. The plates were then washed again as described above. The substrate solution (Neogen Corporation, Lexington, KY, USA, K-Blue TMB substrate) was added ( $100 \mu \mathrm{l}$ per well), and the plate was then incubated for $30 \mathrm{~min}$ on an orbital rotating shaker at 
room temperature. The enzymatic reaction was stopped by the addition of the appropriate solution ( $1 \mathrm{~N} \mathrm{H}_{2} \mathrm{SO}_{4}, 100 \mu \mathrm{l}$ per well), and the absorbance values were read at $450 \mathrm{~nm}$. A cubic regression equation was used to fit the standard curve generated by the ELISA assay. The sample concentration of prostasin was calculated by extrapolation. The inter-assay and intra-assay coefficient of variation of the method were $<9 \%$ and $<11 \%$, respectively; the sensitivity limit of the assay was a prostasin concentration $\leqslant 0.5 \mathrm{ng} \mathrm{ml}^{-1}$. Calibration curves were fairly good, and recovery tests indicated high performance (in terms of $\%$ recovery both in buffer or in urine matrices).

\section{Urinary exosomes: isolation and analysis}

Urine from six normotensive individuals was collected at two different time points during the day (early and late in the morning) and processed for exosome extraction. Urinary $\mathrm{Na}$ was quantified in the collected urine using a routine method. Urinary exosomes were extracted by means of a nanomembrane concentrator procedure following the protocol described by Cheruvanky et al. $^{18}$ with minor modifications. In brief, after extensive vortexing, urine samples were centrifuged at $13000 \times \mathrm{g}$ for $40 \mathrm{~min}$ at $4^{\circ} \mathrm{C}$. Twenty-five milliliters of supernatant were loaded into phosphate-buffered saline preconditioned, sterile nanomembrane concentrators (Vivaspin concentrators, Sartorius, Goettingen, Germany) and centrifuged at $3500 \mathrm{~g}$ and $4{ }^{\circ} \mathrm{C}$ until all the urine was filtered. The flow through was discarded. Exosomes within the filters were recovered using $2 x$ Laemmli buffer.

The quality of the microvesicle preparations was assessed by electron microscopy and Aquaporin western immunoblotting (see Supplementary material). The protein concentrations were estimated using a bicinchoninic acid assay.

\section{Sodium dodecyl sulfate-polyacrylamide gel electrophoresis and western blotting}

Urinary exosome fractions were used for prostasin determination using a specific antibody. Aliquots with the same total protein content $(25 \mu \mathrm{g})$ were loaded onto $12 \% \mathrm{~T}$ sodium dodecyl sulfate-polyacrylamide gels. The gels were run and then transferred to a polyvinylidene difluoride membrane as previously described..$^{10}$ Briefly, the membranes were probed with a prostasin primary antibody (BD Biosciences, Milan, Italy) and anti-Aquaporin 2 primary antibody (Sigma-Aldrich, St Louis, MO, USA) at the appropriate dilutions (1:250 and $1: 2000$ ) in $1 \%$ non-fat dried milk, $0.05 \%$ Tween-20 Tris-buffered saline for $3 \mathrm{~h}$ at room temperature. The blots were then incubated $45 \mathrm{~min}$ at room temperature with the appropriate horseradish peroxidase-conjugated secondary antibody (1:10000 dilution; GE Healthcare Europe GmbH, Milan, Italy). The immunocomplexes were detected by chemiluminescence (ECL, GE Healthcare Europe $\mathrm{GmbH}$ ). The membranes were stained with Amido Black Stain for normalization purposes, and the films were scanned and processed using Quantity One software version 4.4 (GelDoc, Bio-Rad, Hercules, CA, USA).

\section{Biochemical analysis}

The patient blood samples were collected after an overnight fast. All of the biochemical tests, including urinary creatinine, were performed in the Laboratory of Clinical Chemistry of the University of Verona. Aldosterone and direct active renin levels were determined using commercially available methods (DiaSorin Diagnostics, Turin, Italy) and expressed in $\mathrm{pg} \mathrm{ml}^{-1}$.

\section{Statistical analysis}

Statistical analyses were performed with SPSS 16.0 for Windows (SPSS, Chicago, IL, USA). The quantitative values were expressed as means \pm s.d. When necessary, logarithmic transformation was applied to obtain a normal distribution, and the variability of the data were expressed as $95 \%$ confidence intervals. Student's $t$-test for paired data were performed by comparing data from the same subjects. Correlations were tested by simple bivariate correlation analysis.

\section{RESULTS}

In normotensive individuals $(n=58)$, urinary prostasin levels presented a wide range of values (range $0.5-18.9 \mathrm{~nm}$ ) with no differences related to gender (means $3.89 \pm 4.38 \mathrm{~nm}$ in males, $n=28$ and
$5.19 \pm 5.18 \mathrm{~nm}$ in females, $n=30)$. Urinary prostasin levels were positively correlated with the aldosterone to renin ratio (ARR; $r=0.363, P=0.02$ ) but not with circulating aldosterone or renin levels individually (data not shown). No statistical correlation was evident with other biochemical parameters.

Considering the group of 58 healthy individuals as a whole (43 healthy volunteers of both genders and 15 women before OC therapy), all subjects were classified according to the distribution percentiles of prostasin in order to evaluate the specific features associated with different concentrations of the serine protease. The most increased urinary concentration (the upper tertile of the distribution) was associated with both the highest ARR and the highest excretion of prostasin per unit of $\mathrm{Na}$ (Table 1). With the exception of slightly higher serum potassium levels, no other relevant differences were observed within the subgroups with a low-intermediate concentration of prostasin (Table 1).

As aldosterone-mediated $\mathrm{Na}$ reabsorption is strongly influenced by dietary $\mathrm{Na}$ intake and assuming that urinary $\mathrm{Na}$ may serve as a proxy of such intake, possible associations between this latter parameter and urinary prostasin were also investigated. Overall, the concentration of the serine protease was correlated with $\mathrm{U}-\mathrm{Na}$ in a complex way, as described by the plot reported in Figure 1. In summary, the urinary prostasin level increased linearly up to the concentration limit of approximately $200 \mathrm{mmoll}^{-1} \mathrm{Na}$ (below this value, both variables were linearly correlated, with $R=0.40, P<0.05)$, whereas it decreased for higher U-Na concentrations (Figure 1).

To confirm the obtained urinary data, natriuresis-associated changes of 'cell' prostasin were also investigated in urinary exosomes from six different healthy subjects (three men and three women). The urinary exosome quality was assessed by electron microscopy and AQ2 western immunoblotting (Supplementary material). To evaluate the possible inter-individual variations, prostasin immunoblotting was performed in the same individual on exosomes collected twice during the morning (Figure 2). As observed in whole urine, the exosome prostasin concentration was also higher in subjects with higher natriuresis (U-Na values $<200 \mathrm{mmoll}^{-1}$ ), whereas it was decreased when U-Na levels were $>200 \mathrm{mmoll}^{-1}$.

Table 1 Characteristics of healthy subjects according to urinary prostasin excretion

\begin{tabular}{|c|c|c|c|}
\hline & $\begin{array}{c}\text { U-prostasin } \\
\text { Two lower tertiles } \\
(\mathrm{n}=39)\end{array}$ & $\begin{array}{c}\text { U-prostasin } \\
\text { Higher tertile } \\
(\mathrm{n}=19)\end{array}$ & P-value \\
\hline Age & $30.8 \pm 7.3$ & $27.7 \pm 7.9$ & NS \\
\hline $\mathrm{P}-\mathrm{Na}^{\mathrm{b}}\left(\mathrm{mmol}^{-1}\right)$ & 139.7 (139-140.3) & $139.8(139.3-140.3)$ & NS \\
\hline $\mathrm{P}-\mathrm{K}^{\mathrm{C}}\left(\mathrm{mmol}^{-1}\right)$ & $3.821 \pm 0.255$ & $4.05 \pm 0.3$ & $<0.05$ \\
\hline $\mathrm{U}-\mathrm{Na}^{\mathrm{c}}\left(\mathrm{mmol}^{-1}\right)$ & $131 \pm 64$ & $141 \pm 52$ & NS \\
\hline $\mathrm{U}-\mathrm{K}^{\mathrm{C}}\left(\mathrm{mmol}^{-1}\right)$ & $50.2 \pm 25$ & $55 \pm 22$ & NS \\
\hline $\mathrm{U}-\mathrm{Na} / \mathrm{K}^{\mathrm{c}}$ & $2.82 \pm 1.14$ & $2.92 \pm 1.4$ & NS \\
\hline Aldosterone $^{\mathrm{c}}\left(\mathrm{pg} \mathrm{ml}^{-1}\right)$ & $138 \pm 71$ & $158 \pm 75$ & NS \\
\hline $\operatorname{Renin}^{b}\left(p g m l^{-1}\right)$ & $7.8(6.2-9.9)$ & $7.58(5.6-10.3)$ & NS \\
\hline $\mathrm{ARR}^{\mathrm{b}}$ & $18.3(14.4-23.2)$ & $21.3(14.8-30)$ & $<0.05$ \\
\hline U-prostasin/U-Nab & $0.0122(0.010-0.015)$ & $0.056(0.041-0.076)$ & 0.000 \\
\hline
\end{tabular}

Abbreviations: ARR, aldosterone to renin ratio ( $\mathrm{pg} \mathrm{ml}^{-1} / \mathrm{pg} \mathrm{ml}^{-1}$ ); $\mathrm{Cl}$, confidence interval $\mathrm{K}$, potassium; Na, sodium; NS, nonsignificant; U-prostasin/U-Na, ratio between urinary prostasin concentration in nм and urinary $\mathrm{Na}$ in $\mathrm{mmol}^{-1}$

Features of the healthy subjects group taken as a whole $(n=58 ; 43$ healthy volunteers of both genders and 15 women before OC therapy) according to the value of urinary prostasin expressed in tertiles (the highest tertile vs. the others)

a Lower prostasin tertiles $=$ urinary prostasin $<2.8 \mathrm{~nm}$.

bSkewed variables, for which data are geometric means $(95 \% \mathrm{Cls})$

'Data are means \pm s.d. 


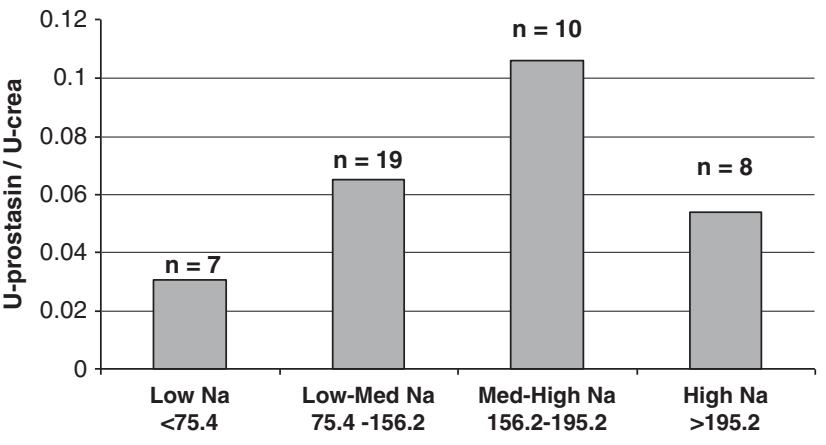

Figure 1 Relationship between urinary (U)-prostasin concentration (nм) and $\mathrm{U}-\mathrm{Na}\left(\mathrm{mmoll}^{-1}\right)$ in healthy subjects. Urinary prostasin increases linearly up to the concentration limit of approximately $200 \mathrm{mmol} \mathrm{I}^{-1}$ sodium ( $\mathrm{Na}$ ) and decreases for more elevated U-Na concentrations. A full color version of this figure is available at the Hypertension Research journal online.

In fertile women, the comparison between the follicular and luteal phases confirmed the activation of the aldosterone-renin axis during the menstrual cycle, with raised luteal aldosterone and renin concentrations but without concordant modifications of their ratio (aldosterone range $167 \pm 90$ to $341 \pm 175 \mathrm{pg} \mathrm{ml}^{-1}, P<0.01$; renin range $12 \pm 8.3$ to $20 \pm 12.4 \mathrm{pg} \mathrm{ml}^{-1}, P=0.05$; ARR range $17.4 \pm 11.3$ to $20.7 \pm 11, P=\mathrm{NS})$. Similar to the ARR, the urinary prostasin level did not substantially vary in the luteal compared with the follicular phase (prostasin range $2.97 \pm 3.58$ to $2.35 \pm 1.23 \mathrm{~nm}$; data not shown).

After OC therapy, the aldosterone and renin values were significantly reduced (aldosterone range $341.0 \pm 175.5$ to $201.0 \pm$ $99.7 \mathrm{pg} \mathrm{ml}^{-1}$; renin range $19.7 \pm 12.5$ to $6.9 \pm 3.3 \mathrm{pg} \mathrm{ml}^{-1}$ ), whereas the ARR increased (from $20.7 \pm 11.0$ to $33.7 \pm 18.3$ ). Urinary prostasin also tended to increase $(2.37 \pm 1.27$ to $4.85 \pm 5.28 \mathrm{nM})$ consistently with the ARR (see above); however, this increase was not statistically significant $(P=0.07$; Table 2$)$.

\section{DISCUSSION}

An important result of this study is the quantitative evaluation of urinary prostasin by ELISA, an assay able to provide a more precise definition of the extent of serine protease release in urine compared with the semiquantitative information provided by previous works that adopted immunoblotting methods.

The ELISA assay was based on prostasin recognition by antibodies specifically reacting toward an immunogenic peptide of prostasin, resulting in a good cross-reaction with the entire recombinant protein used as a standard reference in preliminary experiments and in calibration curves. The accuracy of the method was fairly good, with intra- and inter-assay coefficient of variations $<11 \%$ and a lower detection limit of $\leqslant 0.5 \mathrm{ng} \mathrm{ml}^{-1}$ of prostasin. The method described here is a modification of the assay previously published by Koda et al. ${ }^{11}$ and exploits the same process of production, identification and purification of the same anti-prostasin antibodies.

Only two previous papers, by Koda et al. ${ }^{11}$ and Zhu et al., ${ }^{12}$ have quantitatively measured the concentration of prostasin in the urine of human subjects. In the report by Koda et al., ${ }^{11} 26$ healthy normotensive subjects were evaluated together with 121 hypertensive patients. Thus, the elucidation of the physiological mechanisms regulating the release of urinary prostasin was not easily inferable, considering that $\mathrm{Na}$ renal handling in hypertensive patients is likely to be different from that of normotensive subjects.

Zhu et al. ${ }^{12}$ aimed to evaluate the natriuretic response to a well-defined stress test in black adolescents, but the prost- asin concentration was not measured before the beginning of a $\mathrm{Na}$-controlled diet. Therefore, as was the case with the Koda group, the experimental design did not allow the researchers to draw conclusions on the physiological regulation of prostasin released in urine in response to the natriuresis variations.

The subjects selected for this study were healthy normotensive individuals who were on a free diet, had urinary ions levels compatible with the standard western diet and presented normal aldosterone and renin concentrations. In this context, we were interested in clarifying how urinary prostasin concentration changed in relation to physiological natriuresis in order to gain insights into the natural mechanisms controlling the release of prostasin in urine.

Most of the available findings from in vitro cell culture and animals studies $^{6,19}$ support the viewpoint that prostasin detachment from the glycosylphosphatidylinositol (GPI) anchor represents an obligatory step and/or the consequence of the proteolytic cleavage of activating ENaC. In humans, it has never been demonstrated that prostasin release in vivo is linearly reflected by the functional activation of $\mathrm{ENaC}$. In a rodent model, adenovirus-mediated human prostasin gene delivery was followed by increased urinary $\mathrm{Na}$ excretion despite aldosterone stimulation and BP elevation. ${ }^{19}$ These results were in contrast with the expected $\mathrm{Na}$ retention.

The results of this study provide experimental evidence that the physiological relationship between ENaC activity and urinary prostasin is bimodal and that prostasin is negatively associated with $\mathrm{U}-\mathrm{Na}$ (as theoretically expected) only in high $\mathrm{Na}$ intake conditions. The release of urinary prostasin increased linearly up to the concentration limit of approximately $200 \mathrm{mmoll}^{-1} \mathrm{U}-\mathrm{Na}$, whereas it decreased above this U-Na threshold (see Figures 1 and 2). The phenomenon was observed in a stable condition of normotension, that is, adequate renal $\mathrm{Na}$ handling and $\mathrm{BP}-$ natriuresis relationships. Assuming a direct proportionality with prostasin release in urine, these results suggest that $\mathrm{ENaC}$-mediated $\mathrm{Na}$ reabsorption is physiologically inhibited only at U-Na concentrations over $200 \mathrm{mmol}$, whereas it still occurs at lower concentrations. Taking into account the necessary caution because of the different experimental approaches, the results of this study are also in agreement with the in vitro phenomenon known as 'selfinhibition of ENaC', where intracellular $\mathrm{Na}$ has been shown to inhibit $\mathrm{ENaC}$ cleavage and to thus reduce $\mathrm{Na}$ transport. ${ }^{20}$

Moreover, our previous results obtained using a different approach, based on western immunoblotting, indicate a decrease in urinary prostasin in normotensive individuals after $\mathrm{Na}$ loading and pharmacological aldosterone blockade. ${ }^{10}$ Considering the semiquantitative nature of these results, we are performing further experiments to repeat and confirm our earlier results in a larger set of samples. In addition, our preliminary ELISA data on urinary prostasin (normalized by urinary creatinine) are in agreement with the observed decreasing trend (data not shown).

To further support our results, we studied urinary exosomes as possible 'direct' cellular sources of prostasin. Exosomes are released into the urinary compartment through a process of fusion of the multivesicular body outer membrane with the apical plasma membrane. The exosomal expression of prostasin was consistent with the results obtained from total urine (Figure 2), that is, prostasin increased with a U-Na concentration lower than $200 \mathrm{mmoll}^{-1}$ and decreased for higher concentrations. Taking into account the obvious limitation of an immunoblotting method in terms of quantification and the limited number of subjects, these results confirm that prostasin is finely modulated by urinary $\mathrm{Na}$ on the membranes of the tubular cells. 

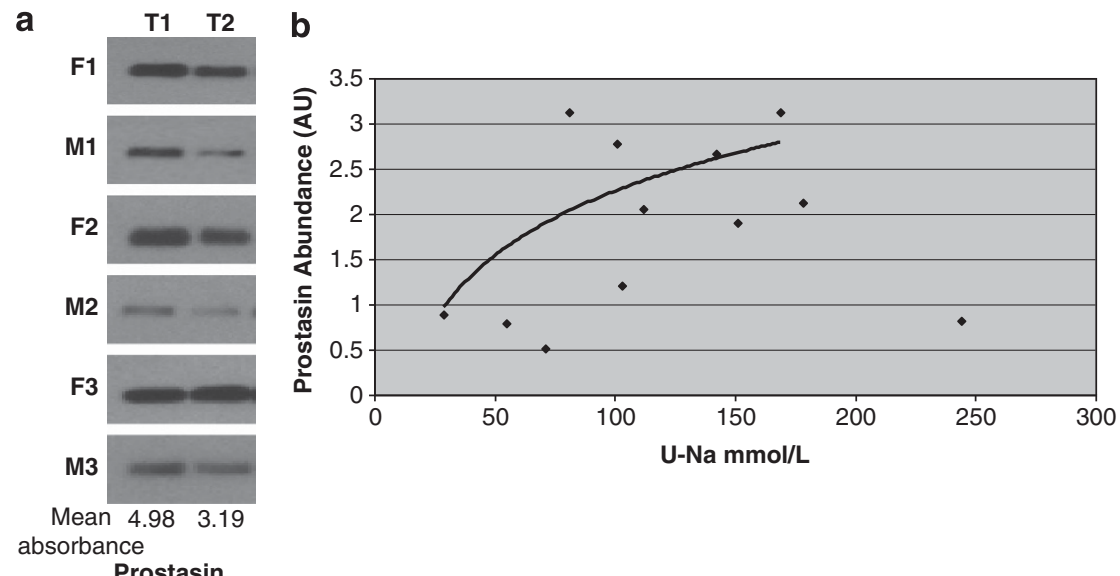

absorbance
Prostasin

Figure 2 Western immunoblotting (a) and graphical representation of urinary sodium (U-Na) excretion (b) according to exosomal prostasin band intensities. At U-Na> $200 \mathrm{mmol}^{-1}$, the prostasin values dropped. The values for prostasin were extracted from exosome prostasin immunoblotting at two time points during the morning in six subjects. An equal amount of total urinary exosomal protein extract was loaded per lane $(25 \mu \mathrm{g})$, and triplicate experiments were performed. AU, arbitrary units. A full color version of this figure is available at the Hypertension Research journal online.

Table 2 Features of healthy women $(n=15)$ before and after OC therapy

\begin{tabular}{|c|c|c|c|}
\hline & $\begin{array}{c}\text { Before OC } \\
\text { therapy }\end{array}$ & $\begin{array}{c}\text { After OC } \\
\text { therapy }\end{array}$ & P-value \\
\hline $\mathrm{SBP}(\mathrm{mm} \mathrm{Hg})$ & $108 \pm 7.7$ & $114 \pm 11.5$ & 0.05 \\
\hline DBP (mm Hg) & $70 \pm 5.6$ & $73 \pm 7.7$ & NS \\
\hline Aldosterone (pg ml-1) & $341.0 \pm 175.5$ & $201.0 \pm 99.7$ & 0.01 \\
\hline Renin (pg ml-1) & $19.7 \pm 12.5$ & $6.9 \pm 3.3$ & 0.002 \\
\hline ARR & $20.7 \pm 11.0$ & $33.7 \pm 18.3$ & 0.001 \\
\hline P-K (mmol I-1) & $3.79 \pm 0.3$ & $3.85 \pm 0.2$ & NS \\
\hline U-Na (mmol I-1) & $108.8 \pm 44$ & $146.0 \pm 34.8$ & 0.014 \\
\hline U- prostasin (nм) & $2.41 \pm 1.27$ & $4.85 \pm 5.28$ & 0.07 \\
\hline $\begin{array}{l}\text { U- prostasin/U-Na } \\
\left(\text { пм per } \mathrm{mmol} \mathrm{I}^{-1} \text { ) }\right.\end{array}$ & $0.023 \pm 0.014$ & $0.033 \pm 0.03$ & NS \\
\hline
\end{tabular}

Data are means $\pm s . d$

Abbreviations: ARR, aldosterone to renin ratio $\left(\mathrm{pg} \mathrm{ml}^{-1} / \mathrm{pg} \mathrm{ml}^{-1}\right)$ ); DBP, diastolic blood pressure; K, potassium; Na, sodium; NS, nonsignificant; OC, oral contraceptive; SBP, systolic blood pressure; U-prostasin/U- $\mathrm{Na}=$ ratio between urinary prostasin concentration in nM and urinary $\mathrm{Na}$ in $\mathrm{mmoll}^{-1}$

The second point of interest in the work stems from the observation that a portion of the normotensive individuals excreted more prostasin, either as absolute concentration or expressed per $\mathrm{Na}$ unit, despite the presence of normal total natriuresis, a normal U-Na/ $\mathrm{U}-\mathrm{K}$ ratio and normal aldosterone and renin concentrations. The only feature peculiar to those subjects was an increased ARR, which reflects the set point of the functional state of the renin-angiotensinaldosterone system. Indeed, while the individual components of the system (aldosterone, renin) may vary in different ways and to different extents, their reciprocal relationship should remain relatively constant under physiologically regulated conditions (that is, such factors as $\mathrm{Na}$ and potassium intake, dehydration, hypervolemia and circadian variation). Interestingly, normotensive individuals with higher levels of prostasin per $\mathrm{Na}$ unit also presented a higher concentration of aldosterone per renin unit. This suggests the likely need to speed up the system in order to maintain the Na equilibrium. It is intriguing to observe that an increased ARR has been indicated as a reliable predictor of subsequent hypertension in prospective studies on normotensive populations. ${ }^{21,22}$ It could be speculated that under hypertensive and pathological renal $\mathrm{Na}$ handling conditions, characterized by increased $\mathrm{ARR}$ and elevated $\mathrm{Na}$ intake, urinary prostasin might also be relatively elevated or not sufficiently inhibited. However, there was ARR overlap between the low and high tertiles of prostasin, so no cutoff value can be set. Therefore, the relationship linking the renin-angiotensin-aldosterone axis, $\mathrm{Na}$ and prostasin in hypertensive individuals remains to be elucidated.

Consistent with previous results, ${ }^{10}$ prostasin was present in the urine of all the subjects without significant differences related to gender. This result strengthens the hypothesis that prostasin is constitutively released in human urine regardless of the potential interference derived from the prostate gland. This result is not secondary as it supports the likelihood that urinary prostasin may serve as a marker of renal origin without confounding contributions from alternative sources.

In healthy women, the prostasin level, unlike the aldosterone level, did not substantially change following the menstrual cycle phases. Therefore, although sexual hormones fluctuations are indeed able to affect aldosterone and renin concentrations, the release of prostasin in urine does not appear to be sufficiently sensitive to these variations or, speculatively, $\mathrm{ENaC}$ activity does not actually change in a significant way.

Estro-progestinic therapy had a greater capability to alter such a balance. In fact, despite mild changes in the aldosterone and renin levels (both decreased), the ARR and the urine concentration of prostasin increased, but the increases did not reach statistical significance. Moreover, although the BP values did not reach pathological levels, they increased substantially after OC therapy and accompanied a significantly increased U-Na.

In conclusion, in healthy subjects, urinary prostasin is similarly present in both genders, and it is not affected by the different phases of the menstrual cycle. Prostasin is modulated by urinary $\mathrm{Na}$, and prostasin levels appear to be correlated with the ARR rather than with individual aldosterone or renin levels. Although a urinary excess of prostasin seems to be associated with ARR, which is the hallmark of conditions such as low-renin hypertension and primary aldosteronism, the precise relationship linking the renin-angiotensinaldosterone axis, $\mathrm{Na}$ and prostasin in hypertensive patients deserves further investigation.

\section{ACKNOWLEDGEMENTS}

The measurement of prostasin concentration was performed with the assistance of Hitachi Chemical. This work was supported by a grant from the Italian Ministry for University and Scientific Research. 
1 Alvarez de la Rosa D, Canessa CM, Fyfe GK, Zhang P. Structure and regulation of amiloride-sensitive sodium channels. Ann Rev Physiol 2000; 62: 573-594.

2 Hamm LL, Zhuang F, Hering-Smith KS. Regulation of sodium transport by ENaC in the kidney. Curr Opin Nephrol Hypertens 2010; 19: 98-105.

$3 \mathrm{Yu} \mathrm{JX}$, Chao L, Chao J. Prostasin is a novel human serine proteinase from seminal fluid. Purification, tissue distribution, and localization in prostate gland. J Biol Chem 1994; 269: 18843-18848.

4 Vallet V, Chraibi A, Gaeggeler HP, Horisberger JD, Rossier BC. An epithelial serine protease activates the amiloride-sensitive sodium channel. Nature 1997; 389: 607-610.

5 Adachi M, Kitamura K, Miyoshi T, Narikiyo T, Iwashita K, Shiraishi N, Nonoguchi H, Tomita K. Activation of epithelial sodium channels by prostasin in Xenopus oocytes. J Am Soc Nephrol 2001; 12: 1114-1121.

6 Narikiyo T, Kitamura K, Adachi M, Miyoshi T, Iwashita K, Shiraishi N, Nonoguchi H, Chen LM, Chai KX, Chao J, Tomita K. Regulation of prostasin by aldosterone in the kidney. J Clin Invest 2002; 109: 401-408.

7 Sheng S, Carattino MD, Bruns JB, Hughey RP, Kleyman TR. Furin cleavage activates the epithelial sodium channel by relieving $\mathrm{Na}+$ self-inhibition. Am J Physiol Ren Physiol 2006; 290: 1488-1496.

8 Kitamura K, Tomita K. Regulation of renal sodium handling through the interaction between serine proteases and serine protease inhibitors. Clin Exp Nephrol 2010; 14: 405-410.

9 Chen LM, Skinner ML, Kauffman SW, Chao J, Chao L, Thaler CDE, Chai KX. Prostasin is a glycosylphosphatidylinositol-anchored active serin protease. J Biol Chem 2001; 276: 21434-21442.

10 Olivieri O, Castagna A, Guarini P, Chiecchi L, Sabaini G, Pizzolo F, Corrocher R, Righetti PG. Urinary prostasin, a candidate marker of epithelial sodium channel activation in humans. Hypertension 2005; 46: 683-688.

11 Koda A, Wakida N, Toriyama K, Yamamoto K, Iijima H, Tomita K, Kitamura K. Urinary prostasin in humans: relationships among prostasin, aldosterone and epithelial sodium channel activity. Hypertens Res 2009; 32: 276-281.
12 Zhu H, Chao J, Guo D, Li K, Huang Y, Hawkins K, Wright N, Stallmann-Jorgensen I, Yan W, Harshfield GA, Dong Y. Urinary prostasin: a possible biomarker for renal pressure natriuresis in black adolescents. Pediatr Res 2009; 65: 443-446.

13 Oelkers W. Effects of estrogens and progestogens on the renin-aldosterone system and blood pressure. Steroids 1996; 61: 166-171.

14 Pizzolo F, Raffaelli R, Memmo A, Chiecchi L, Pavan C, Guarini P, Guidi GC, Franchi M, Corrocher R, Olivieri O. Effects of female sex hormones and contraceptive pill on the diagnostic work-up for primary aldosteronism. J Hypert 2010; 28: $135-142$.

15 Ma X, Fu Y, Li Y, Chen L, Chai K, Wang Y. Prostasin inhibits cell invasion in human choriocarcinomal JEG-3 cells. Histochem Cell Biol 2009; 132: 639-646.

16 Pisitkun T, Shen R-F, Knepper MA. Identification and proteomic profiling of exosomes in human urine. Proc Natl Acad Sci USA. 2004; 101: 13368-13373.

17 van der Lubbe N, Jansen PM, Salih M, Fenton RA, van den Meiracker AH, Danser AH, Zietse R, Hoorn EJ. The phosphorylated sodium chloride cotransporter in urinary exosomes is superior to prostasin as a marker for aldosteronism. Hypertension 2012; 60: 741-748.

18 Cheruvanky A, Zhou H, Pisitkun T, Kopp JB, Knepper MA, Yuen PS, Star RA. Rapid isolation of urinary exosomal biomarkers using a nanomembrane ultrafiltration concentrator. Am J Physiol Renal Physiol 2007; 292: F1657-F1661.

19 Wang $\mathrm{C}$, Chao J, Chao L. Adenovirus-mediated human prostasin gene delivery is linked to increased aldosterone production and hypertension in rats. Am J Physiol Regul Integr Comp Physiol 2003; 284: 1031-1036.

20 Knight KK, Wentzlaff DM, Snyder PM. Intracellular sodium regulates proteolytic activation of the epithelial sodium channel. J Biol Chem 2008; 283: 27477-27482.

21 Newton-Cheh C, Guo C-Y, Larson MG, Benjamin EJ, Wang TJ, Kathiresan S, O’Donnell CJ, Musone SL, Camargo AL, Drake JA, Levy D, Hirschhorn JN, Vasan RS. Clinical and genetic correlates of aldosterone to renin ratio and relations to blood pressure in a community sample. Hypertension 2007; 49: 846-856.

22 Meneton P, Galan P, Bertrais S, Heudes D, Hercberg S, Ménard J. High plasma aldosterone and low renin predict blood pressure increase and hypertension in middleaged Caucasian populations. J Hum Hypertens 2008; 22: 550-558.

Supplementary Information accompanies the paper on Hypertension Research website (http://www.nature.com/hr) 